\title{
Who Hath Measured the (Proving) Ground: Variation in Offensive Capabilities Test and Evaluation
}

\author{
JD Work \\ Marine Corps University, Quantico, VA \& Columbia University, New York, NY \\ jw3646@columbia.edu \\ DOI: 10.34190/ICCWS.20.086
}

\begin{abstract}
Multiple recent high-profile cyber intrusion and attack incidents have demonstrated serious weaponeering failures in which offensive tooling has not performed as the operators, planners, and designers had likely anticipated, leading to detection, degraded mission outcomes, and political blowback. These failures may be evaluated as resulting from multiple factors, in part through engineering errors introduced within the development lifecycle, as well as from immature command \& control (C2), planning and the operational oversight processes. These cases suggest that despite the different identified failure modes in capabilities generation and employment, a common root cause of operational blunders may be identified in the lack of effective controlled range testing of exploit and implant capabilities packages prior to fielding and use in the wild. Observed evidence to date strongly indicates multiple intrusion sets pursue only limited-and in some case-no validation measures prior to executing live fires against target systems and networks. We seek to describe and explain apparent variations in adoption of munitions effectiveness testing for cyberweapons. We examine requirements, objectives, and benefits of capabilities validation efforts, balanced against resource investment, organizational integration, process agility, operational responsiveness, and other costs. We propose a model for analysis of mission assurance contributions provided by the cyber proving ground and consider this model in light of specific observed adversary behaviors indicating programmatic practices. We further explore the implications for the employment of such validation measures as a fundamental element of developing norms for responsible state cyber operations.
\end{abstract}

Keywords: offensive cyber operations, exploitation, targeting, weaponeering, cyber range

\section{Introduction}

Messenger:

"My lord high constable, the English lie within

fifteen hundred paces of your tents."

Constable:

"Who hath measured the ground?"

Shakespeare. Henry V. Act 3, Scene 7

Consideration of offensive cyber capabilities often relies on analogies to more traditionally understood weapons systems. While the framing and conceptualization of the "cyber weapon" remains problematic in some international relations and other academic literature, there is an undeniable utility in the consideration in this light of certain tooling offering malicious functionality to compromise the confidentiality, integrity, and availability of targeted systems and networks (Peterson 2013, Repel and Hersee 2015, Kello 2017, Schmidle Sulmeyer and Buchanan 2017, Smeets 2018, Prunckun 2018, Rid 2018). For this reason, the conceptualization of the cyber weapon will continue to persist - and with it attending consideration of the functions involved in developing, acquiring, sustaining, and employing a given capability as a weapon in the offensive context.

Beyond this utility, understanding offensive cyber capabilities framed as weapons allows one to bring to bear further lenses that may be drawn from a longstanding academic and professional literature examining innovation in armaments construction and use, strategies of deployment and positioning, and other broader questions involved in examining the sociology of weapons (Jacobson 1987, Flank 1993, MacKenzie 1993, Eden 2004, Kristensen and Norris 2012, Centeno and Enriquez 2016).

Core among the processes of weapons employment is the function of weaponeering, defined as part of the targeting cycle. In doctrinal terms, weaponeering may be thought of in the narrow sense of determining allocation of the inevitability limited munitions resources to achieve deliberate effects objectives (DOD 2019). However, the original focus on quantity presumes a specific and binary effect from munitions delivery (e.g. kinetic explosion). As this concept broadened to encompass assessment of target vulnerability, delivery accuracy, damage criteria, and weapon reliability as well as indirect outcomes from higher order effects and 


\section{JD Work}

other nonkinetic effects, the complexity of the tasks involved further matured into the full scope function of capabilities analysis. This in turn influences the closely related functions of force assignment in current and future operations planning.

The classified nature of specific cyber munitions effects assessment has largely precluded more detailed discussion of these processes to date, but joint staff planners have acknowledged that cyber operations could be incorporated into existing targeting workflow with only minor adaptation (Williams 2014). These processes in Western services also remain new, with initial prototype development of a long sought Cyber Joint Munitions Effectiveness Manual (JMEM) by the US Department of Defense Joint Technical Coordinating Group for Munitions Effectiveness announced only in 2016 after more than a decade of development, which in turn incorporated US Cyber Command's Cyber Capabilities Registry as well as the Electronic Warfare and Cyber Critical Elements Weaponeering Guides (Gallagher and Horta 2013, DOD 2016).

Cyber weapons present unique considerations beyond the scope of traditional kinetic and even psychological effects planning. In part, this is due to the uniquely mutable nature of offensive cyber capabilities, where effects may be tailored as part of targeting and operational planning in ways that conventional munitions generally may not. And although one may consider the analogy of "dial-a-yield" full fuzing option nuclear warhead designs, the planning considerations of nuclear weapons employment under operational circumstances is a rarified enough field of endeavor that experience with variable effects weapons is atypical (Putz and Nelson 1979).

However, ongoing commercial cyber threat intelligence and other case reporting now offers insights into adversary and unattributed third party action that may support further study and analysis of these activities by a broader range of researchers. From such case examples, this paper offers analysis of incidents in which offensive cyber planners and operators failed to conduct adequate weaponeering, resulting in unintended outcomes including detection and collateral damage. The prospective role of cyber weapons assessment under controlled detonation conditions against threat representative targets is then considered as a potential control against such failure modes, while the factors that may constrain such operational test and evaluation as part of hostile intrusion campaigns are outlined. The paper provides new contributions to the literature on offensive cyber operations, with implications for the ongoing study of capabilities maturity, counterproliferation, and responsible state behavior.

\section{Weaponeering failure cases}

The immaturity of adversary programmatic structures for test and evaluation of offensive cyber operations capabilities have been observed in multiple high profile cases in recent years. In these instances, the delivery of effect against a target system or network did not occur in the manner that one would expect to see in a robust concept of operation, suggesting strongly that these effects were the result of unintended consequences arising out of previously unidentified failure conditions. These conditions may be assessed as stemming from weaponeering failure, in that the wrong capability was deployed against the target - likely in no small part due to the fact that intrusion planners and operators did not understand the performance characteristics of the exploit and / or implant package, and the associated leadership structure responsible for directing the offensive activity failed to exercise appropriate oversight at relevant times in the lifecycle of the operation. We may see this in both well known incidents where these factors have been, to date, largely overlooked, as well in as in lesser publicized cases that have not yet received substantial analytic scrutiny.

\subsection{Stuxnet}

Among the earliest prominent failure may be seen in the uncontrolled propagation of the offensive capability payload commonly known as Stuxnet. This case has been described extensively in the cyber conflict analysis literature as well as popular media. Despite this publicity, there is a high likelihood that the extensive gaps in the public understanding of critical operational details renders many conclusions regarding the activity suspect due to the many unknowns of the campaign. However, one salient point is at this point understood. At the time of global detection in the summer of 2010, a specific variant of the weapon had spread laterally from infected target networks to other networks more rapidly, and far more widely, than the formally unattributed planners likely anticipated. This propagation failure almost certainly derived from unintended developer error in managing the malware's exploitation logic, coupled with what may have been less robust degree of review on these factors due to developer assumptions regarding the intended nature of the deployment environment 
- no doubt presumed to be air-gapped and tightly controlled. The Stuxnet platform incorporated multiple nested exploit chains targeting then zero-day vulnerabilities in the target operating systems, intended to trigger against a likely range of potential system configurations that may have been encountered in the course of delivery as the payload sought to spread autonomously from initial infection beachhead laterally across target networks in order to reach specific operational technology against which the payload was designed to deliver deliberate effects. The introduction of a single exploit option, targeting the then widespread CVE-20084250 vulnerability subsequently patched by MS08-067, is believed to have produced extensive unintended lateral movement where the payload was delivered - although additional controls incorporated within the code ensured that no effects against non-targeted systems were executed, and in fact the malware further automatically removed itself from non-targeted systems.

The decision process for selection of the specific exploit options utilized in the disclosed variant is unclear, but was likely informed by contemporary intelligence regarding the target ecosystem. Prior widespread exploitation of CVE-2008-4250 vulnerabilities by the criminal Conficker worm had resulted in extensive challenges for many cybersecurity professionals seeking to secure legacy RPC functionality found in the Windows operating system and associated software architectures. The coordinated takedown of Conficker botnet infrastructure, and sinkholing of victim communications with Conficker command and control (C2) servers, had produced substantial insight into vulnerable systems known to have been previously compromised through this exploit, and which likely remained unpatched (Bowden 2011). Commercial cyber threat intelligence reporting is known to have previously leveraged this insight in order to characterize the information environment of Iranian leadership and associated military interests, and identified the potential for use of this type of applied vulnerability intelligence for targeting in offensive cyber operations (iSIGHT Partners 2009). Significantly, this analysis was almost certainly provided to the US government as part of acknowledged ongoing contract support for cyber intelligence missions (Stone 2016). Such findings, whether as here reported by industry or in similar parallel but unknown targeting analysis efforts, may further explain previous unsupported allegations made by some security researchers that Conficker infections may have served as an initial entry point for later Stuxnet operations, despite the clear original criminal intent and pattern of life observed in the Conficker botnet activity (Finkle 2011).

The extensive engineering evidenced within the Stuxnet capability package is widely believed to suggest a high degree of mission assurance effort had been pursued by developers and planners (Freedberg 2012, Rosenbaum 2012). Media reporting further suggests that the payload was tested within a threat representative target network custom built for this purpose (Sanger 2012). Nonetheless, error apparently surfaced in part due to divergence between expected conditions as understood by planners and developers involved in design and configuration of the weapon's autonomous logics, and the actual conditions found within targeted network where initial access foothold had been obtained. The nature of this divergence remains unclear due to the limited public reporting regarding early phases of the campaign - with some suggestions of human-enabled close access operations, as well as associated supply chain infection vectors (Zetter 2014, Murchu 2014, Zetter and Modderkolk 2019). Each of these scenarios differ by which propagation restraints would have failed, and from these scenarios it may be inferred that not all potential access conditions and subsequent staging options had been appropriately modeled in capabilities test and evaluation - suggesting a potential later-stage change in development after initial review. One further cannot rule out potential changes in concept of operations, or change in execution due to conditions encountered in the field after development and "containerization" of the implant and delivery package.

However, the number of unknown unknowns in this case as reported to date, coupled with the inability to independently evaluate key facts underpinning alleged narrative points derived solely from uncertain transmission through purportedly highly classified government information leaks, render this example of limited value in more generalized inductive inference beyond serving to outline the problem space.

\subsection{ETERNALBLUE in Wannacry and Nyeta malware}

In other cases, adversary development has also incorporated exploit packages in a manner that failed to deliver controlled execution against intended targets. Two major incidents illustrate these dynamics well, emerging within weeks of each other. On 14 April 2017 an unattributed actor calling itself the "Shadowbrokers" publicly released code for multiple exploit portfolios and implant tooling which the group alleged to have acquired by undescribed means from a purported National Security Agency offensive cyber operations program. The group, active since the late summer of 2016, is widely suspected of having been a 
misattribution front operating on behalf of Russian intelligence services (Loleski 2016, FireEye 2017a). Among the leaked capabilities was an exploit targeting Microsoft operating system Server Message Block (SMB) protocol implementation vulnerabilities CVE-2017-0144 / CVE-2017-0145 patched by MS17-010. This exploit was circulated under the purported cover term ETERNALBLUE (Microsoft 2017, Brown 2017).

At this time, an ongoing intrusion set known as APT38 / HIDDEN COBRA / LAZARUS / Hermit / STARDUST CHOLLIMA, attributed by industry to the North Korean intelligence services, had reportedly been developing a new ransomware variant intended to continue illicit revenue generation operations in support of regime leadership objectives as part of ongoing cyber crime campaigns utilizing multiple theft and extortion tactics. Early variants of the malware which came to be known commonly as Wannacry sought to propagate independently through abuse of insecure SMB protocol configurations. Industry researchers observed continuing iterated development of this tool in apparent limited releases in the wild for a period of some months. After the Shadowbrokers leak, the North Korea developers rapidly integrated the improved ETERNABLBLUE SMB lateral movement option into the Wannacry malware (Symantec 2017, CrowdStrike 2017ab). The resulting weapon was deployed through prior botnet infrastructure maintained by the HIDDEN COBRA / LAZARUS actor on 12 May 2017 (CrowdStrike 2017c, FireEye 2017b). Although this release was perhaps not intended for full operational scale, due to limited development of associated monetization infrastructure needed to realize profits from ransomware extortion demands, the payload nonetheless spread globally with substantial disruptive and destructive effects (FireEye 2017c).

Similarly, autonomous lateral movement options had also previously been leveraged in malware delivered by a Russian origin intrusion set frequently targeting systems and networks in the Ukraine in connection with the ongoing conflict in the country. This intrusion set, attributed by industry under various cryptonyms SANDWORM / VOODOO BEAR / IRIDIUM / ELECTRUM and associated GreyEnergy subset, had previously executed multiple destructive cyber attacks leveraging both wiper malware, as well as ransomware deployed to deliver nonreversible effects. In December 2016, the campaign reportedly tested a new ransomware variant dubbed Moonraker Petya against a small number of live targets, delivering limited destructive effects not widely reported at the time. The malware implant was a tool almost certainly acquired from the cyber criminal underground market, and modified by the adversary operators for their own purposes. This evidence was consistent with prior acquisition efforts by the group which had leveraged other crimeware and open source tooling for low cost, rapid capabilities generation further offering utility in deception for misattribution objectives, including attempts to mask destructive payloads as ransomware observed as early as January 2017 (ESET 2017a, FireEye 2017d). SANDWORM / VOODOO BEAR / GreyEnergy operators likewise rapidly incorporated the leaked ETERNALBLUE exploit into further capabilities development activities, likely sometime after 20 May 2017 when apparent developmental testing of an intermediate spiral variant was observed. (CrowdStrike 2017b). The new weaponized package, dubbed Nyeta / NotPetya by industry, was deployed on 27 June 2017 (CrowdStrike 2017cde, FireEye 2017e, Palo Alto 2017). Initial infections were delivered via a previously staged supply chain compromise providing large scale access to Ukrainian critical infrastructure and business networks through a mandatory accounting software package that taxpayers in the country were required to use by law (Cisco 2017, CrowdStrike 2017f, ESET 2017b). Delivering fires through supply chain vectors may have been intended to limit effects to targets in Ukraine, however the autonomous spread of the malware across flat networks of multinational business entities whose subsidiaries operated in country rapidly led to a global outbreak inflicting substantial collateral damage.

In both cases, it is clear that adversary developers recognized the ETERNALBLUE's utility for penetration of target systems, but failed to appropriately consider capability behavior in lateral movement across real-world network conditions. The fully engineered exploit was almost certainly beyond the developers' independent discovery capacities, and was apparently grafted onto extant propagation logic that did not take into account wormable scenarios. It is further apparent that operators lacked intelligence in advance of deployment against specific target environments, relying instead on a generalized (and as it turned out, highly flawed) mental model of a typical enterprise system likely drawn from personal experience rather than deliberate reconnaissance.

\subsection{XENOTIME / Veles intrusion set deployment of TRISIS / TRITON / HATMAN malware}

Another major case emerged in the summer of 2017, resulting from sustained intrusion activity targeting oil and gas critical infrastructure in Saudi Arabia. Malware attributed to the XENOTIME / Veles activity group intended to alter integrity of safety instrumentation systems was deployed within the operational technology 
networks controlling physical plant processes at a targeted facility. The intrusion, associated by industry with Russian intelligence service contractor operations, was ultimately detected due to unexpected behavior of the targeted Tristation controller (Orleans 2018, FireEye 2019). The attackers had moved laterally within the compromised enterprise network through manually interactive methods using common systems administration tools repurposed for malicious purposes, in the process defeating multifactor authentication countermeasures. However, the actors attempted to deliver a custom weaponized payload for effects against the facility's distributed control systems network, dubbed by commercial researchers TRITON / TRISIS / HATMAN. This tool had been developed with features suggesting specific advance knowledge of the targeted safety systems, requiring prolonged access to the relevant equipment. Despite this, initial attempts to exercise control over targeted nodes failed - likely due to a combination of factors involving differing software versions, memory states, and initial configurations. Multiple attempts to deliver malware against the node resulted in device failure, denying future access options for the specific subsystem and alerting facility owners and defenders (Dragos 2017, FireEye 2018ab, Slowik 2018).

In short, while adversary actors likely had substantial intelligence support - potentially derived from suspected access to the targeted victim's own network for over three years prior to the attempted actions on objective an apparent lack of prior testing at adequate fidelity resulted in a botched operation.

\section{Capabilities validation through controlled test \& evaluation}

A distinction may be made in the noted cases between operator error and planning failure. In the former instances, mere friction may be considered part of the inescapable nature of warfare in any domain, to be mitigated to the extent possible through training, operator excellence, and line management. In the latter cases, one may observe that the adversary lacked knowledge of the weapon, the conditions of its deployment, and had not adequately considered its probable effects across the target environment. This demonstrates the utility provided by a priori test and evaluation of new offensive cyber capabilities. Such evaluation should be conducted under controlled conditions, much as in any other research and development effort for a new weapons system. In common US and allied practice, testing is a key function resulting in extensive investment in cyber range facilities intended to serve both operator training purposes but also as a testbed for new effects.

The cyber proving ground serves as the first opportunity to render tangible abstractions of the coding process by which offensive capabilities are developed. While the best developers will have had prior offensive experience - even if only in playing Red Team roles in education or exercises - this experience is not a prerequisite nor should be automatically assumed. Even where coder talent may have enjoyed prior such practice, there is a vast difference between simple capture the flag games and more substantive simulation of reconnaissance, intrusion and attack functions. Many otherwise good programmers will thus find themselves tasked with engineering tasks for which they lack a robust mental model of the target, the context in which a capability will be deployed against that target, and the conflict environment in which the target is situated.

Management structures providing guidance and direction to these developers may fare little better, particularly where the rapidly changing pace of technology adoption, altered missions and new objectives may render obsolete even those with more substantive prior work histories. These gaps may be overcome by robust intelligence support and close collaboration of designers with planners and operators. However, such interactions may be complicated by considerations of secrecy and compartmentalization - particularly involving the classes of offensive cyber operations conducted under covert action authorities.

Yet even in tightly coupled multi-functional teams, the fundamental character involved in executing malicious instructions within targeted compute architectures is grounded in finding and leveraging unexpected, unknown or previously unimagined conditions through which one may pursue arbitrary authentication, access, privilege, and manipulation outside of the intended parameters of systems as designed. It is inevitable that whilst grappling with the complexity of interactions across attack surfaces, control flow, boundary conditions, versioning / patching and associated mitigations, that developers and operators will encounter situations that could not have been modeled or foreseen. In fact, there is a growing recognition at the cutting edge of offensive security that while functional exploitation has been delivered in practice for decade, there remain deep uncertainties regarding the theoretical framework for reasoning about exploitation. This in turn impacts the ability to estimate future (non)exploitability under differing conditions (Dullien 2017). The lack of robust 
frameworks within computer science to explain the character and performance of these "weird machine" states imposes basic and often underappreciated risks in the evaluation of effects, reliability in delivery of these effects, and control of collateral or otherwise unanticipated outcomes as one moves from engineering to warfighting applications.

It is where munition meets metal (or at least its virtualized instantiation and hypervisor) that the assumptions of design, development and planning are tested. In the repetition of this impact under varying conditions one may gain confidence in the reliability of an offensive capability. Even more critically it is here one may curate the scenarios under which reliability has been tested in order that these may be communicated to the planners, operational management, and oversight functions who will be responsible for decisions regarding the employment of these capabilities. Accurate evaluation is predicated on threat representative targets, including specific versioning, configuration, and implementation characteristics that may be unique to each potential adversary environment and even within different elements of the same adversary network, and which frequently change over time. This creates demand for detailed tactical and technical intelligence regarding these targets, and as a corollary prioritizes the sorts of modular tooling and flexible development efforts that may adapt given capabilities to the widest range of potential target specifics. Fidelity in assessment also requires that test scenarios are closely tailored to align with concepts of operations and employment, as uncertainty increases where gaps exist. While these factors pose challenges for offensive programs seeking to hoard capabilities against the widest range of possible future needs, conversely the limitations of reserve magazine depth over time in part may be overcome by stockpile assurance efforts enabled by range operations within a wider arsenal management framework - in effect giving new longevity to capabilities that might otherwise have been deprecated, based on findings from the proving ground (Work 2018).

\section{Adversary constraints at the proving ground}

However, it is clear that adversary operators continue to forgo such controlled testing, executing operations either with only limited quality assurance evaluation or even as has been seen testing through initial live fire cases. Multiple factors likely contribute to these recurring decision patterns.

A number of threat programs have essentially developed from bootstrapped capabilities - that is, a combination of criminal and open source software repurposed for espionage and cyber attack objectives. More sophisticated programs may have also matured through emulation, including the capture and replay of payloads and mimicry of observed tactics, techniques and procedures. In these types of programs, the investment into organic capabilities generation has been more limited - and therefore it is unlikely that associated mature functions of quality assurance and operational test and evaluation under controlled conditions would be pursued in the face of an apparent developer and management preference for acquisition of new capabilities versus further development of older tooling. In these cases, decisionmakers may not even be aware of the true origins of a given capability, with industry reporting suggesting multiple instances in which adversary acquisition efforts merely "reskinned" a publicly available tool through localization and other minor modifications, in patterns familiar from illicit market transactions (ESET 2017b, FireEye 2014abc, Cherepanov \& Lipovsky 2016, CCDP 2014).

Many adversary capabilities may be further characterized by predominately individual effort, where limited talent is left alone to pursue objectives under relatively loose direction. These patterns may be partly explained by the independent and ego-investment driven behaviors of many hacker communities from which key talent is recruited, trained and further developed. Individual efforts may also be reflected in a preference for manual interactive intrusion actions, where operators on the keyboard make immediate decisions in direct engagement, rather than executing a pre-planned course of action decided by planners and approved by leadership. Lack of management guidance or effective oversight may also be the result of disconnects across levels of organization, where seniors fail to understand or appreciate the realities "in the trenches." Such failures have notable historical parallels, including the perceived "Chateaux generals" of World War I (Nuño 2018).

Task preferences may also be reflected, based on variations in prestige and competition for work roles within cyber operations elements. Offensive activities are commonly seen to demand greater talent, and are more readily recognized by leadership, in connection with perceptions of elite status. On the other hand, building 


\section{JD Work}

and running a range environment for test and evaluation functions is frequently seen as a more basic system administration function, thus a supporting role. In these instances, the talent assigned may be ill-fit or unprepared for purpose. As a corollary, individual dwell time in such assignments may be therefore insufficient to develop needed expertise regarding appropriate scenario definition, test protocols, and assessment criteria as specific operators seek to move to other, higher prestige "real world" roles as quickly as they can rotate. In turn, this costs experience and credibility at the programmatic level, further reducing the likelihood of future work towards understanding offensive capability effectiveness and constraints prior to fires in the wild. These disconnects may also degrade potential effectiveness of proving ground activities, where validation through operational test \& evaluation (OT\&E) is treated merely as a checklist requirement rather than an opportunity for collaboration to improve cyber weapons delivery and effects.

Even where cyber range environments may have been stood up and appropriately staffed, munitions effectiveness may be evaluated only against what are truly threat representative targets. Merely adequate effort towards building only generic architectures at only small scale will often be insufficient to uncover the edge cases and boundary conditions where operational problems develop. Many adversary programs may be forced to work only with nonrepresentative targets at varying degrees of known loss of fidelity due to unavailability of better options in the face of limited budgets, sanctions pressures, inability to validate normal business transactions to acquire target equipment (particularly in tightly knit commercial communities involved in sales of relatively rare operational technologies). Changing enterprise deployment and technology sector business models have further exacerbated these dynamics, as targets move onto cloud infrastructure and other increasingly uniquely custom environments that pose exceptional difficulties for replication on the range using only commodity compute and networking resources. And even if adequate resources may be marshalled, specific intelligence is needed to match target specific configuration and work patterns. The dynamics here may serve as explanatory factors in recent increasingly active campaigns assessed as pursuing espionage against critical infrastructure networks. These factors also point to future counter-proliferation mission requirements, wherein denying adversary access to such key enabling elements will greater drive risk of adversary operational failure and associated uncertainty for leadership that might therefore be more reluctant to approve future operations, and where options may exist to impose friction on adversary capabilities generation through counter-cyber operations and other active measures.

\section{Implications and Outlook}

Failures in contact, influenced by multiple factors described herein, have illustrated that there are substantial consequences from the nonuse of cyber range environments as proving grounds to validate offensive cyber capabilities prior to deployment in the wild - leading to negative outcomes risking a greater probability of detection, more severe blowback, and associated global condemnation for excessive collateral damage. These failures also contribute to the ever more strained characterizations of "covert" action in cyberspace, in which the (im)plausible deniability of ambiguous or deliberately deceptive misattribution has been increasingly of limited value to adversary leadership seeking to escape these consequences due to high profile events resulting from bad design, poor planning and disastrous weaponeering judgements. As a result, it is likely that greater maturity in offensive operational practices will be sought by these actors, under pressure from management and leadership. Such maturity will also likely been seen as well in the emerging new entrants who may learn from these cases as they too seek to develop their own offensive programs.

It is further clear that there are limitations in addressing only the most well-known recent cyber incidents of significance. Whilst features of these cases were discussed that had previously escaped consideration, it is quite likely that the routine behavior of adversary planners and operators under more ordinary conditions rather than the moments of dramatic effort and associated failure - may yield even greater insight into the offensive cyber praxis. One may anticipate therein the opportunity to derive a deeper understanding of the interplay between intelligence, reconnaissance and operational preparation of the environment activities, and associated options design and development for later cyber effects operations. Likewise, while the cases here considered encompassed weaponeering as a means of preventing unanticipated effects and collateral damage, further research may illuminate the connection between cyber munitions delivery and mechanisms of indirect and higher order effect - including perhaps filling critical gaps in our understanding of leadership (mis)perceptions following intrusion or attack incident detection and disclosure that may lead to improperly interpreted signaling, emotional and other responses from cognitive traps, and ultimately the risk of inadvertent escalation. 


\section{JD Work}

It is in this latter space that we may find the most productive potential for such future research. The evolution of norms around offensive cyber operations has been slow, if not entirely stunted (Eggenschwiler and Silomon 2018) Decisionmakers ultimately provide the limit stakes for intelligence and military service activities under their direction, and if we are to see the development of mature norms setting expectations of responsible state behavior in cyber conflict, the strategic policy level must be connected to the working technical and tactical front in a manner that developers, planners, and virtual "trigger pullers" can understand and implement on a repeatable basis. Lessons learned from specific cases will be required to educate operating forces and to inculcate the principles of responsibility and restraint needed in order to ensure a more stable and secure cyberspace into the future.

\section{References}

BOWDEN, M. 2011. Worm: The First Digital World War, London, Atlantic Books. BROWN, R. "The Shadow Brokers Leaked Exploits Explained". Rapid7. 18 April

DEPARTMENT OF DEFENSE. 2016. FY16 Live Fire Test and Evaluation (LFT\&E). UNCLASSIFIED. https://www.dote.osd.mil/pub/reports/FY2016/pdf/other/2016lfte.pdf

---. 2019. Joint Publication 3-69: Targeting.

CENTENO, M. A. \& ENRIQUEZ, E. 2016. War \& Society, Cambridge, UK, Polity Press.

CISCO TALOS. 2017. "The MeDoc Connection". 5 July

CROWDSTRIKE. 2017a. "Identified Wanacry Ransomware Predecessor and Potential DPRK Overlap". 16 May

---. 2017b. "Three WanaCry Ransomware Variants Identified; Code Overlaps with Intrusion Set Attributed to North Korean Actor STARDUST CHOLLIMA". 16 May

---. 2017c. "Mass Distribution of Wana Ransomware Causes Significant Disruption" 12 May

----. 2017d. "Identified Wanacry Ransomware Predecessor and Potential DPRK Overlap". 16 May

---. 2017e. "Early Stand-Alone Variant of Petya-Based Wiper".

---. 2017f. "Large-Scale Ransomware Attack: Spreading Strategy Combines ETERNALBLUE Exploit and SMB Credential Stealing". 27 June

---. 2017g. "Technical Analysis of PetrWrap". 29 June

---. 2017h. "Additional Analysis Suggest Petya-Based Ransomware Campaign was Designed as Targeted Destructive Wiping Attack". 29 June

---. 2017i. "Malicious M.E.Doc Software Updates Used To Deliver Destructive Payloads". 14 July

CHEREPANOV, A. \& LIPOVSKY, R. 2016. BlackEnergy: what we really know about the notorious cyber attacks. Virus Bulletin Conference. Denver, Colorado, USA.

CYBER CONFLICT DOCUMENTATION PROJECT (CCDP). 2014. "Field Note: Early popularity of Poison Ivy malware among Chinese hackers". 23 March

DRAGOS. 2017. "TRISIS Malware: Analysis of Safety System Targeted Malware". 13 December

DULLIEN, T. F. 2017. Weird machines, exploitability, and provable unexploitability. IEEE Transactions on Emerging Topics in Computing.

EDEN, L. 2004. Whole World on Fire: Organizations, Knowledge, and Nuclear Weapons Devastation, Ithaca, NY, Cornell University Press.

EGGENSCHWILER, J. \& SILOMON, J. 2018. Challenges and opportunities in cyber weapon norm construction. Computer Fraud \& Security, 11-18.

ESET. 2017a. "GREYENERGY: A successor to BlackEnergy". October

ESET. 2017b. "Analysis of TeleBots' cunning backdoor". 4 July

FINKLE, J. 2011. Insight: Did Conficker help sabotage Iran program. Reuters, 2 December.

FIREEYE. 2014a. "'Dendroid' Android RAT Most Likely a Modification of Dehydroid RAT; Probably Has Growing User Base". 18 March

---. 2014b. "Medusa Grabber Likely Rebranded Version of Carbon Form Grabber". 21 March

---. 2014c. "'Development of njwOrm, H-Worm and the Derivation of Similar Remote Access Trojan Variants Through the Arabic-Language Underground". 11 September

---. 2017a. "Group Profile: Shadow Brokers". 20 October

---. 2017b."Highly Prolific WannaCry Ransomware Campaign". 18 May

---. 2017c. "WannaCry Malware Likely Connected to North Korean Actors; Authority Behind Campaign Unclear". 25 May

---. 2017d. "Pattern of Previous Activity and Limited Technical Indicators Support Sandworm Group Responsibility for Petya Campaign". 30 June

---. 2017e. "Assessing Attribution and Intent Behind the Global Petya Ransomware Incident". 29 June

---. 2018a. "A Totally Tubular Treatise on TRITON and TriStation". 7 June.

---. 2018b. "'TRITON: Comprehensive Analysis of the Attack Lifecycle from Intrusion to Attack Execution". 19 October

---. 2019. "TRITON Attribution: Russian Government- Owned Lab Most Likely Built Custom Intrusion Tools for TRITON Attackers". 11 February

FLANK, S. 1993. Exploding the Black Box: The Historical Sociology of Nuclear Proliferation. Security Studies, 3:2, 259-294.

FREEDBERG, S. J. Jr. 2012. Cyber Command Lawyer Praises Stuxnet, Disses Chinese Cyber Stance. Breaking Defense, 12 March. 


\section{JD Work}

GALLAGHER, M. A. \& HORTA, M. C. 2013. Cyber Joint Munitions Effectiveness Manual (JMEM). American Intelligence Journal, 31, 73-81.

iSIGHT PARTNERS. 2009. Assessing the Persian blogosphere's impact on the information environment of the Iranian Parliament.

JACOBSEN, C. G. 1987. The uncertain course: New weapons strategies and mind-sets, Oxford, Oxford University Press. KELLO, L. 2017. The Virtual Weapon, New Haven, CT, Yale University Press.

KRISTENSEN, H. M. \& NORRIS, R. S. 2012. Nonstrategic nuclear weapons. Bulletin of the Atomic Scientists, 68:5, 96-104. LOLESKI, S. 2019. From cold to cyber warriors: the origins and expansion of NSA's Tailored Access Operations (TAO) to Shadow Brokers. Intelligence and National Security, 34:1, 112-128.

MACKENZIE, D. A. 1993. Inventing Accuracy: A Historical Sociology of Nuclear Missile Guidance, Cambridge, MIT Press. MICROSOFT. 2017. "Protecting customers and evaluating risk. 14 April. https://msrcblog.microsoft.com/2017/04/14/protecting-customers-and-evaluating-risk/

NUNO, G. 2018. Chateau Cyber: Applying Historical Events to Military Innovation in the Cyber Domain. In 13th International Conference on Cyber Warfare and Security (ICCWS) p. 427.

ORLEANS, A. 2018. Bears in the Lines: Russian Critical Infrastructure Espionage in the Face of US Grid Resilience. CYBERWARCON. Arlington, VA.

PALO ALTO NETWORKS. 2017. "Threat Brief: Petya Ransomware". 27 June

PETERSON, D. 2013. Offensive Cyber Weapons: Construction, Development, and Employment. Journal of Strategic Studies, $36: 1,120-124$

PUTZ, D. W. \& NELSON, D. B. 1979. Series II tests of B77 component section. Sandia Labs.

REPEL, D. \& HERSEE, S. 2015. The Ingredients of Cyber Weapons. 10th International Conference on Cyber Warfare and Security. Kruger National Park, South Africa.

ROSENBAUM, R. 2012. Richard Clarke on Who Was Behind the Stuxnet Attack. Smithsonian Magazine.

SANGER, D. E. 2012. Confront and Conceal: Obama's Secret Wars and Surprising Use of American Power, New York, Random House.

SCHMIDLE, R. E. Jr., SULMEYER, M. \& BUCHANAN, B. 2017. Nonlethal Weapons and Cyber Capabilities. In: PERKOVICH, G. \& LEVITE, A. E. (eds.) Understanding Cyber Conflict: Fourteen Analogies. Washington, DC: Georgetown University Press.

SMEETS, M. 2018. A matter of time: On the transitory nature of cyberweapons. Journal of Strategic Studies, 41:1-2, 6-32.

STONE, J. 2016. Meet the Cyber-Industrial Complex: Private Contractors May Get \$7B Windfall From Pentagon's Cyberwar On ISIS. International Business Times, 7 March.

SYMANTEC. 2017. "WannaCry: Ransomware attacks show strong links to Lazarus group". 22 May

WILLIAMS, B. T. 2014. The Joint Force Commander's Guide to Cyberspace Operations. Joint Force Quarterly, 73.

WORK, J.D. 2019. Calculating the Fast Equations: Arsenal management considerations in sustained offensive cyber operations. Cambridge, MA: Cyber Security Project, Belfer Center for Science and International Affairs, John F. Kennedy School of Government, Harvard University.

ZETTER, K. \& MODDERKOLK, H. 2019. Revealed: How a secret Dutch mole aided the U.S.-Israeli Stuxnet cyberattack on Iran. Yahoo News, 2 September.

ZETTER, K. 2014. Countdown to Zero Day, New York, Crown Publishers. 